\title{
Niosomal drug delivery for transdermal targeting: recent advances
}

This article was published in the following Dove Press journal:

Research and Reports in Transdermal Drug Delivery

29 July 2015

Number of times this article has been viewed

\section{Rita Muzzalupo \\ Lorena Tavano}

Department of Pharmacy and Health and Nutrition Science, University of Calabria, Edificio Polifunzionale, Cosenza, Italy
Correspondence: Rita Muzzalupo Dipartimento di Farmacia e Scienze della Salute e della Nutrizione, Università della Calabria, Edificio Polifunzionale, 87036 Arcavacata di Rende, Cosenza, Italy Email rita.muzzalupo@unical.it
Abstract: Niosomes are vesicular nanocarriers and are receiving much attention as potential transdermal drug delivery systems due to properties such as enhanced drug penetration, local depot for sustained drug release, and a rate-limiting membrane for modulation of systemic absorption of drugs via the skin. Several mechanisms have been proposed to explain the ability of niosomes to increase drug transfer through the skin. Niosomal carriers are suitable for the transdermal delivery of numerous pharmacological agents, including antioxidant, anticancer, anti-inflammatory, antimicrobial, and antibacterial molecules, and this review attempts to provide an exhaustive collection of recent investigations in this interesting field, with special emphasis on the strategies used to enhance the potential of niosomes.

Keywords: niosomes, transdermal, permeation, enhancer, drug delivery

\section{Introduction}

This review provides a brief overview of issues related to niosomes by explaining their chemical composition, structure, advantages, and applications, makes general remarks on niosomes as percutaneous permeation enhancers, and discusses the findings of investigations done over the past 5 years on niosomal drug delivery systems for transdermal applications.

Niosomes are vesicular nanocarriers and have received much attention as potential drug delivery systems in the last 30 years due to their unique advantages. They have lamellar (bilayer) structures composed of amphiphilic molecules surrounded by an aqueous compartment. These amphiphilic molecules, known as surfactants, contain both hydrophobic groups (tails) and hydrophilic groups (heads) and show self-assembling properties, aggregating into a variety of shapes like micelles or into a planar lamellar bilayer. ${ }^{1}$ Surfactants that could be used as potential drug delivery systems include sorbitan esters and analogs, sugar-based, polyoxyethylene-based, polyglycerol, or crown ether-based surfactants, sometimes in addition to membrane additives, such as cholesterol or its derivatives. Nonionic surfactants are preferred because they have less potential to cause irritation, which decreases in order of cationic $>$ anionic $>$ nonionic. $^{2}$

The unique structures of niosomes as vesicular systems make them capable of encapsulating both hydrophilic and lipophilic substances. Hydrophilic drugs are usually encapsulated in the inner aqueous core or adsorbed on the bilayer surfaces, while lipophilic substances are entrapped by their partitioning into the lipophilic domain of the bilayers (Figure 1). 


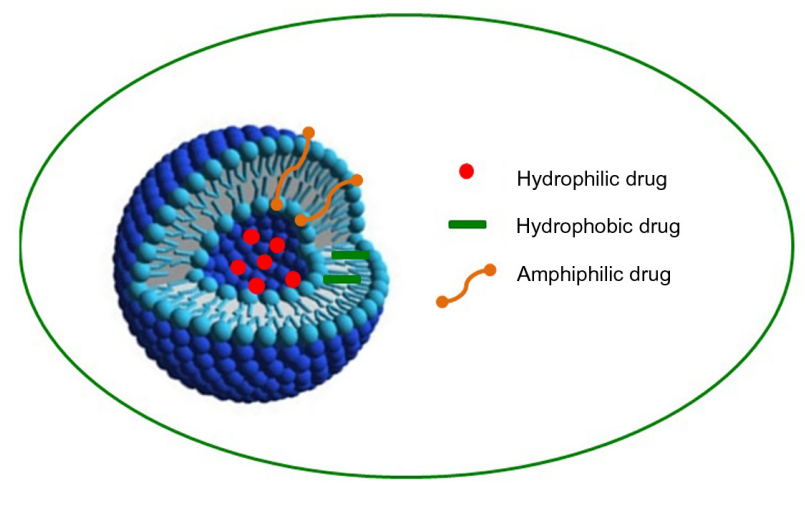

Figure I Schematic representation of a niosomal vesicle.

The formation of vesicular assemblies requires the input of some form of energy, and all the experimental methods investigated involve hydration of a mixture of surfactants above the gel to liquid phase transition temperature of the system, followed by optional size reduction to obtain a colloidal dispersion. ${ }^{3}$ Because of their potential ability to carry a variety of therapeutics, these vesicles have been widely used as drug delivery systems to achieve drug targeting, controlled release, and permeation enhancement. ${ }^{4}$ In fact, niosomes can act as therapeutic reservoirs for delivery of a drug in a controlled manner to enhance bioavailability, obtaining a therapeutic effect over a longer period of time, and can be modified by altering the composition, concentration of various additives, and surface charge of vesicle components and membrane additives. ${ }^{5}$ Moreover, drug ionization has been found to modulate the physicochemical properties of the vesicles and their percutaneous permeation profiles. ${ }^{6}$ In recent decades, niosomes have been investigated in-depth as potential carriers for sustained and targeted drug delivery, since they are easily derivatized to enhance vesicles versatility to improve the affinity for the target site. ${ }^{?}$

\section{Advantages and disadvantages of niosomal carriers}

Niosomes combine several advantages with respect to other nanocarriers: ${ }^{8}$

- Surfactants used to prepare niosomes are biodegradable, biocompatible, and not immunogenic

- The method used for routine and large-scale production of niosomes does not involve use of unacceptable solvents

- Due to the chemical stability of their structural composition, the handling and storage of niosomes does not require any special conditions
- The physicochemical properties of niosomes, such as their shape, fluidity, and size, can be easily controlled by changing their structural composition and the method of production

- Niosomes are able to encapsulate a large amount of material in a small vesicular volume

- The structure of niosomes protect drug ingredients from heterogeneous factors present both inside and outside the body, so niosomes can be used for the delivery of labile and sensitive drugs

- Niosomes improve the therapeutic performance of drug molecules by delaying clearance from the circulation and restricting effects to target cells

- Niosomes can be administered via different routes, such as oral, parenteral, and topical, and using different dosage forms such as powders, suspensions, and semisolids, improving the oral bioavailability of poorly soluble drugs and also enhancing the permeability of drugs through the skin when applied topically

- The aqueous vehicle-based suspension formulation results in better patient compliance when compared with oily dosage forms; in addition, niosomal dispersion, being aqueous, can be emulsified in a nonaqueous phase to regulate the drug release rate

- Niosomes have been reported to achieve better patient adherence and satisfaction and also better effectiveness than conventional oily formulations.

At the same time, niosomes have some disadvantages, which may decrease their shelf life, and include physical and chemical instability, aggregation, fusion of vesicles, and leaking or hydrolysis of the encapsulated drug. Moreover, the methods required for preparation of multilamellar vesicles, such as extrusion or sonication, are time-consuming and may require specialized equipment for processing. ${ }^{9}$

\section{Niosomes versus liposomes}

Niosomes and liposomes are functionally the same, with similar physicochemical properties depending on the composition of the bilayer and the preparation methods used (Table 1). They act as amphiphilic vesicles, and both can be used for targeted and sustained drug delivery.

Several authors have reported that the function of niosomes in vivo is similar to that of liposomes. ${ }^{10}$ Niosomal and liposomal vesicular systems have similar applications in the pharmaceutical and cosmetic field, but differ chemically in their structure units; niosomes are made of surfactants whereas liposomes are based on phospholipids, meaning that niosomes have greater stability and lack many 
Table I Niosomes versus liposomes: a summary

\begin{tabular}{lll}
\hline & Niosomes & Liposomes \\
\hline Components & Surfactants & Phospholipids \\
Component availability & High & Low \\
Component purity & Good & Variable \\
Preparation and storage & No special & Inert atmosphere and \\
& conditions required & low temperature \\
Stability & Very good & Low \\
Cost & Low & High \\
\hline
\end{tabular}

of the disadvantages associated with liposomes, ie, high cost, low availability, and the variable purity problems associated with phospholipids. Niosomes do not require special conditions such as low temperature or an inert atmosphere during preparation and storage; these features make niosomes more attractive for industrial manufacturing. ${ }^{11}$ On the other hand, niosomes offer several advantages over liposomes, such as intrinsic skin penetration-enhancing properties. ${ }^{12}$

\section{Applications of niosomes}

Niosomes were introduced for use in the cosmetic industry. The first report on surfactant vesicles came from the cosmetic applications devised by L'Oreal. ${ }^{13}$ Phospholipids and nonionic surfactant have been reported to act as penetration enhancers that can overcome the barrier of transdermal drug delivery. ${ }^{14}$ Since then, there has been increasing interest in the use of niosomes in the pharmaceutical, cosmetic, and food industries, leading to the publication of more than 1,200 research articles, about 200 patents, and six clinical trials from 1980 onwards. Most of these publications make reference to the importance of characterization of nanovectors.

Niosomal carriers are suitable for the delivery of numerous pharmacological and diagnostic agents, including antioxidants, anticancer, anti-inflammatory, antiasthma, antimicrobial, anti-Alzheimer's, and antibacterial molecules, oligonucleotides, and others. ${ }^{5}$ Depending on the type of drug, surfactant, disease, and anatomical site involved, various routes of administration exist for niosomal drugs, ie, intravenous, intramuscular, oral, ocular, subcutaneous, pulmonary, and transdermal. ${ }^{4}$ Several other routes have been used to administer niosomal drugs, including the intraperitoneal and vaginal routes. Niosomes have been used for successful targeting of drugs to various organs like the liver and brain or to pathological districts such as tumor, enhancing drugs pharmacological activities while reducing side effects. ${ }^{15}$ In particular, targeted niosomal systems have been designed with different mechanisms of action, including active, passive, and magnetic targeting, leading to more advanced and specific macromolecular drug carriers. ${ }^{16}$

\section{Toxicity of niosomes}

The toxicity of niosomes is related to their components, ie, nonionic surfactants are more biocompatible and less toxic than their anionic, amphoteric, and cationic counterparts. When the same surfactants are in the form of vesicular systems, these properties strongly decrease. There is little research published on the toxicity of niosomes and the types of surfactant included. ${ }^{17}$ Hofland et al ${ }^{18}$ evaluated the toxicity of the types of surfactant used in niosomal formulations to human keratinocytes, and demonstrated that ester types of surfactant are less toxic than ether types due to enzymatic degradation of bonds in esters. Hemolytic tests are traditionally used to predict the toxicity of a surfactant and in vesicular systems derived from them. ${ }^{19}$ Recently, it has been demonstrated that the ability of niosomes to disrupt erythrocytes depends on the length of the alkyl chain in the surfactant and on the size of the colloidal aggregates in solution. Presumably, a shorter carbon chain intercalates better into the membranes of erythrocytes, destructing their molecular organization; niosomes have more difficulty to interact with biological membranes, resulting in substantial hemolysis. ${ }^{20}$ Niosomes prepared with bolaform surfactants showed encouraging safety and tolerability data both in vitro in human keratinocytes and in vivo in human volunteers, who showed no skin erythema when topically treated with a drug-free bolaform niosome formulation. ${ }^{21}$

\section{Niosomes as percutaneous permeation enhancers}

When applying niosomes to the skin, one has to differentiate what type of effect is required, ie, a local effect within the skin (dermal drug delivery) or a systemic effect accompanied by permeation through the skin (transdermal drug delivery), as shown in Figure 2.22

Reaching the bloodstream is the aim of transdermal targeting, and is becoming a focus of interest for many pharmaceutical research groups studying diseases such as inflammation, cancer, psoriasis, alopecia, and acne. ${ }^{11}$ The transdermal route has several advantages over the conventional routes of drug administration: peak and trough levels in serum (a risk and inconvenience of intravenous therapy) are avoided; first-pass hepatic metabolism and gastrointestinal degradation ( $\mathrm{pH}$, enzymatic activity, and interactions with food, beverages, and other orally administered drugs), 


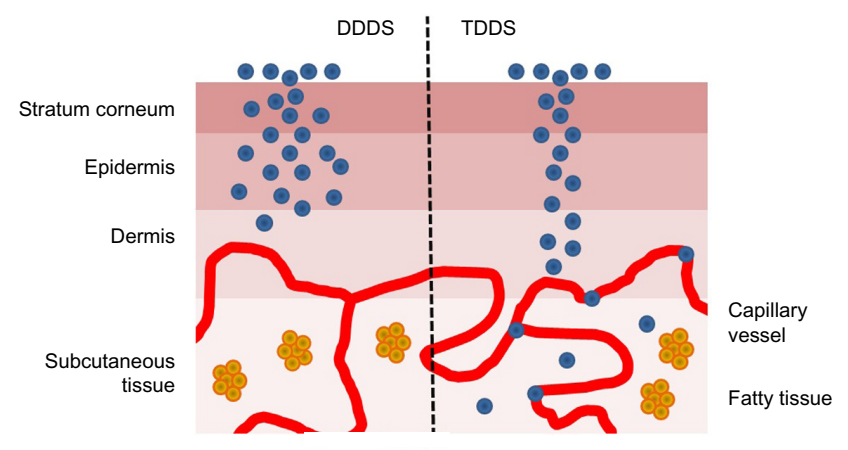

Figure 2 Dermal drug delivery systems and transdermal drug delivery system. Abbreviations: DDDS, Dermal drug delivery systems; TDDS, transdermal drug delivery systems.

are avoided, leading to an increase in drug bioavailability and efficacy; and it can serve as an alternative to oral drug administration when that route is unsuitable (eg, vomiting and diarrhea). Other advantages of the transdermal route include the accessibility of the skin, the relatively large surface area for absorption, and the fact that it is noninvasive, making the patient more compliant.

However, the transdermal route of drug administration has a major disadvantage, ie, a low penetration rate through the skin. Only a limited number of drugs can be formulated as transdermal delivery systems due to the effect of the stratum corneum, which serves as a rate limiting step during drug permeation. This layer is very selective with respect to the types of molecule that it allows to be transported through the skin; therefore, only molecules with specific physicochemical properties can cross the skin adequately. ${ }^{23}$

Drug transfer across the stratum corneum is mainly a passive process and can occur via three routes: intercellular, transcellular (paracellular), and transappendageal. Once it has traversed the epidermis, a compound may be removed by the dermal circulation or be transported to deeper tissues. ${ }^{24}$ Many strategies have been assessed for their ability to overcome the barrier function of the stratum corneum and to improve drug transport into the skin. In particular, penetration enhancers may act by one or more of three potential mechanisms according to the lipid-protein-partitioning theory: they can alter the intercellular lipid structure between the corneocytes to increase diffusivity; and they can modify intracellular protein domains within the horny layer and may increase partitioning of the drug into the skin tissue. ${ }^{25}$ During the last decade, niosomes have undergone intensive investigation for transdermal drug delivery, and seem to be promising vehicles for active substances and targeting to the skin layer. Niosomes are becoming popular in the field of topical drug delivery due to their outstanding characteristics and the properties induced by their presence in a formulation, such as enhanced drug penetration, local depot for sustained drug release and a rate-limiting membrane for modulation of systemic drug absorption via the skin. ${ }^{26}$

An important research contribution to evaluation of niosomal vesicles as a permeation enhancer was made in 2011. The objective of the researchers was to determine if the increased hydrophilic drug permeation across the skin, which is always observed with vesicular systems, is dependent on the structural organization of the niosomes that are used to transport the active molecule or if it is only dependent on the dual nature of surfactant. ${ }^{27}$ Percutaneous permeation profiles of sulfadiazine were obtained by using surfactants both as components of niosomal systems and as submicellar solution, according to the following experimental conditions: treatment with a niosome formulation, a submicellar solution of surfactant, aqueous drug solution after pretreatment of the skin with empty niosomes, and aqueous drug solution after pretreatment of the skin with a submicellar solution of surfactant. The results showed that permeation of sulfadiazine was not increased after pretreatment with a submicellar solution of surfactant or direct treatment with a submicellar solution of surfactant containing the drug respect to the control. Also, pretreatment with empty niosomes followed by treatment with the drug solution showed similar trends, meaning that only direct treatment of the skin with loaded niosomes increased the percutaneous permeation of the drug, confirming the role of niosomes as enhancers. Direct contact between the vesicles and the skin is essential for efficient delivery, although surfactants apparently do not penetrate into the deeper skin layers, and the presence of drug and vesicular carriers must be simultaneous. Only in this case the intercellular lipid barrier in the stratum corneum would be dramatically changed to be more permeable.

\section{Mechanisms of action of niosomes as permeation enhancers}

There is no single mechanism that can sufficiently explain the ability of niosomes to increase drug transfer through the skin, and several mechanisms (Figure 3) have been proposed, including: alteration of the barrier function of the stratum corneum, as a result of reversible perturbation of lipid organization, ${ }^{28}$ reduction of transepidermal water loss, which increases hydration of the stratum corneum and loosens its closely-packed cellular structure; ${ }^{29}$ and adsorption and/or fusion of niosomes on the surface of the skin, as revealed by freeze fracture electron microscopy and small angle X-ray 


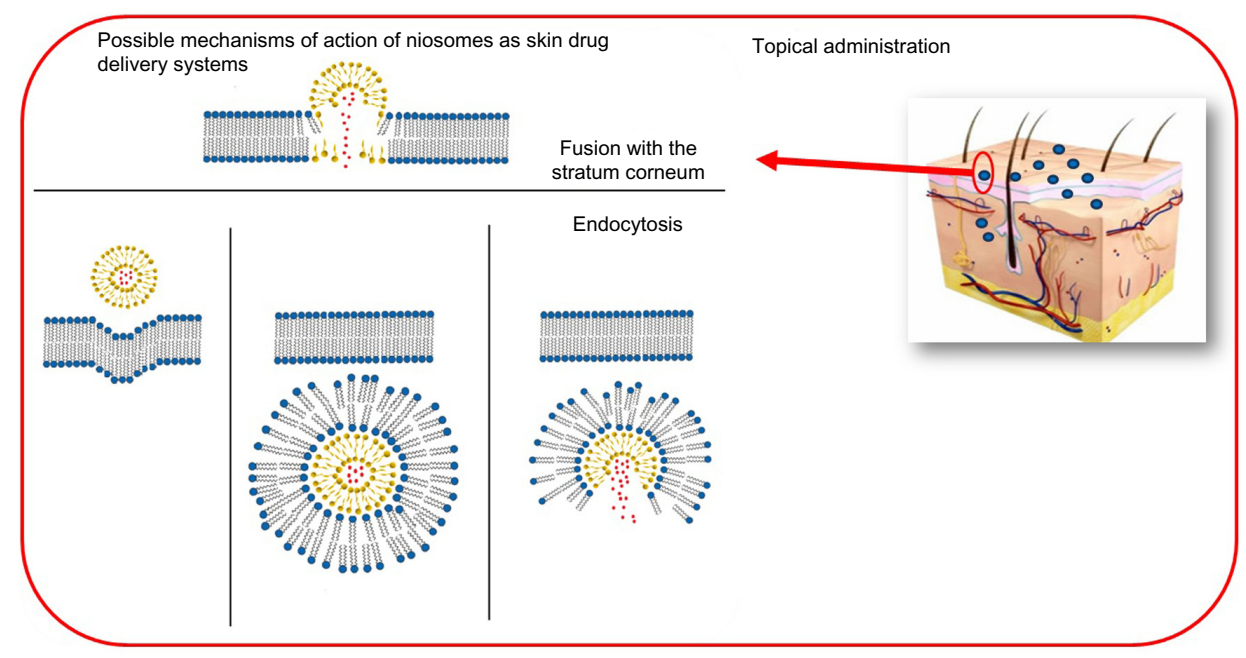

Figure 3 Mechanisms of action of niosomes as skin drug delivery systems.

scattering, leading to a high thermodynamic activity gradient of drug at the interface, which is the driving force for permeation of a drug. ${ }^{30}$

Adsorption of niosomes onto the cell surface occurs with little or no internalization of either aqueous or lipid components; it may take place either as a result of attracting physical forces or as a result of binding by specific receptors to ligands on the vesicle membrane and transfer of drug directly from vesicles to the skin. On the other hand, niosomes may fuse with the cell membrane, resulting in complete mixing of the niosomal contents with the cytoplasm. Finally, niosomes may be engulfed by the cell (endocytosis), with lysozymes present in the cytoplasm degrading or digesting the membranous structure of the niosome, thereby releasing the entrapped material into the medium..$^{31,32}$

\section{Recent advances in niosomal formulations for transdermal drug targeting}

During recent years, transdermal drug delivery from niosomes has been studied in a number of disease models, and current efforts are focused on optimization of procedures, new compositions, and final formulations. For example, new highly flexible niosomes, known as elastic vesicles, have been proposed and are reported to be effective at delivering molecules through the skin, since edge activators (ie, ethanol) provide vesicles with elastic characteristics, which allow them to penetrate more easily into the deeper layers of the skin..$^{33}$ Moreover, the major limitation of niosomes is the liquid nature of the preparation, because when applied they may leak from the application site. This challenge can be overcome by incorporation of niosomes in an adequate vehicle, which can be achieved by adding gelling agents to niosomal dispersions, thereby forming a niosomal gel. ${ }^{34}$ Niosomal gels were found to enhance retention of therapeutics by the skin and to provide high and sustained drug concentrations in the skin. ${ }^{35} \mathrm{~A}$ further evolution of niosomes is represented by proniosomes or "dry niosomes", which have been proposed as niosomal formulations; these need to be hydrated before use, and hydration results in formation of an aqueous niosomal dispersion. Proniosomes decrease the aggregation, leakage, and fusion problems associated with traditional niosomes and offer a versatile transdermal drug delivery system because, upon application to the skin, they become hydrated with water from the skin under occlusion. ${ }^{36}$ A summary of the findings of investigations over the past 5 years for transdermal niosomal drug delivery systems is given in Table 2.

Niosomes containing nonsteroidal anti-inflammatory drugs (NSAIDs) have been prepared by different groups of researchers. These drugs may cause local mucosal irritation and undergo first-pass metabolism in the liver after oral administration, which leads to partial inactivation. Thus, only $50 \%$ of the drug reaches the circulation. Topical dosage forms are desirable for long-term use of this drug, especially when used to treat rheumatic symptoms. The efficacy of topical NSAIDs depends greatly on their capacity to penetrate through the skin.

Nasr et $\mathrm{al}^{12}$ reported better stability and efficacy of their newly developed niosomes when compared liposomes for targeting of aceclofenac via the skin. The anti-inflammatory effect of aceclofenac vesicles was assessed using the rat paw edema technique. The data showed that the entrapment efficiency and in vitro release of aceclofenac from 
Table 2 Summary of recent niosomal formulations developed for drug transdermal delivery

\begin{tabular}{|c|c|c|}
\hline TDDS & Drug & Reference \\
\hline \multirow[t]{8}{*}{ Niosomes } & Aceclofenac & 12 \\
\hline & Diclofenac sodium & $4 I$ \\
\hline & Ketoprofen & 43 \\
\hline & Baclofen & 47 \\
\hline & Salidroside & 48 \\
\hline & Capsaicin & 49 \\
\hline & $\begin{array}{l}\text { Resveratrol, alpha-tocopherol, } \\
\text { curcumin }\end{array}$ & 50 \\
\hline & Alpha-tocopherol & 57 \\
\hline \multirow[t]{7}{*}{ Niosomal gel } & Aceclofenac & 37 \\
\hline & Meloxicam & 38,39 \\
\hline & Rofecoxib & 42 \\
\hline & Simvastatin & 53 \\
\hline & Sulfadiazine sodium, propranolol & 35 \\
\hline & hydrochloride, tyrosol & \\
\hline & Lopinavir & 36 \\
\hline Elastic vesicles & Sulfadiazine sodium & 56 \\
\hline \multirow[t]{2}{*}{ Elastic vesicle gel } & Diclofenac diethylammonium & 40 \\
\hline & Papain & 51 \\
\hline \multirow[t]{4}{*}{ Proniosomes } & Vinpocetine & 42 \\
\hline & Simvastatin & 52 \\
\hline & Nisoldipine & 54 \\
\hline & Nifedipine & 55 \\
\hline \multirow[t]{2}{*}{ Proniosomal gel } & Tenoxicam & 44 \\
\hline & Flurbiprofen & 45 \\
\hline
\end{tabular}

Abbreviation: TDDS, transdermal drug delivery systems.

the vesicles can be manipulated by varying the cholesterol content, the type of surfactant used, and the type of charge. Both vesicular systems had significant sustained antiinflammatory activity when compared with the marketed product, with niosomes being superior to liposomes, as manifested by edema and inhibition percentages, suggesting their effectiveness as topical anti-inflammatory delivery systems.

Aceclofenac niosomes have also been prepared using Span 60 and cholesterol at different molar ratios for topical use after incorporation into carbopol gel..$^{37}$ Data reported by Solanki et $\mathrm{al}^{37}$ showed that the gel improves the penetration and therapeutic efficacy of the drug in all niosomal gel preparations when compared with the plain gel formulation. Moreover, the niosome formulation could act as a reservoir for a prolonged period and serve as a penetration enhancer.

The study reported by El-Menshawe and Hussein ${ }^{38}$ concerns transdermal delivery of meloxicam loaded into niosomes. Their data revealed that niosomes prepared from Span 60 and cholesterol had less side effects and there was a marked increase in inhibition of edema in animals treated with meloxicam vesicular gel when compared with animals treated with free meloxicam.
Nonionic surfactant vesicles have been prepared using Span 60 and cholesterol at different ratios for transdermal delivery of meloxicam as an anti-inflammatory drug. ${ }^{39}$ Three different gel bases were also prepared (using poloxamer-407, chitosan, and carbopol-934) and converted into niosomal gels. The flux of meloxicam was found to be independent of the viscosity of the formulations. The anti-inflammatory effects of meloxicam in the different niosomal gel formulations were evaluated using the carrageenan-induced rat paw edema method, which showed the superiority of niosomal gels over conventional gels.

Manosroi et a ${ }^{40}$ prepared novel elastic niosomes containing entrapped diclofenac diethylammonium, an NSAID, for topical use. Various bilayer vesicular formulations composed of dipalmitoylphosphatidylcholine, Tween 61 , or Span 60 mixed with different molar ratios of cholesterol and ethanol at $0 \%-25 \%(\mathrm{v} / \mathrm{v})$ were prepared. Elastic Tween 61 niosomes, which had advanced physicochemical properties including no sedimentation, no layer separation, and unchanged particle size, were selected to entrap diclofenac diethylammonium. The entrapment efficiency of the drug in the conventional and elastic Tween 61 niosomes was $65 \%$ and $93 \%$, respectively. The deformability index values for the elastic niosomes were markedly higher than for the conventional empty or loaded niosomes, indicating the higher flexibility of the elastic vesicles, especially when the drug was entrapped within the niosomes. Gel containing these elastic niosomes showed flux of diclofenac diethylammonium in the skin greater than that of the commercial Emulgel product, which contain an equivalent amount of drug. The authors demonstrated the enhancement of transdermal absorption through rat skin, and also good anti-inflammatory activity in the rat ear edema assay when diclofenac diethylammonium is entrapped in their novel elastic Tween 61 niosomes.

In 2014, Tavano et $\mathrm{al}^{41}$ investigated the effect of compartmentalization of diclofenac sodium on the physicochemical properties of niosomal vesicles when used as transdermal carriers. Mixtures of Span 60/F127 and Tween 60/F127 at different ratios were used to obtain the niosomes, with the objective of adding new experimental data regarding the influence of the nature of the niosomal matrix on the physicochemical properties of niosomal vesicles and the effect of compartmentalization of diclofenac sodium on drug entrapment efficiency and skin permeability. The results demonstrated that all niosomes were spherical and homogeneous in shape. Their size was found to be dependent on the hydrophile-lipophile balance of the surfactant mixture, ie, an increase in hydrophobicity resulted in smaller vesicles. Drug 
incorporation led to a significant variation in vesicle size which was dependent on the whether the drug was located in the aqueous compartment or the bilayer compartment. In particular, niosomes that had the drug located into the aqueous compartment showed the highest diameter, but when the drug was loaded into the bilayer, the vesicle size decreased slightly, and this trend was more pronounced when the drug was located in both compartments at the same time.

Higher cumulative amounts of diclofenac permeated across rabbit skin after 24 hours were obtained by formulations in which the drug was located in the aqueous core. The data obtained indicate that changing the bilayer composition and drug compartmentalization offers the possibility to modulate the physicochemical properties of the vesicles and drug delivery.

Rofecoxib is another NSAID that has been encapsulated in niosomes. In the work reported by Das and Palei, ${ }^{42}$ niosomes were incorporated into a topical gel base for sustained therapeutic action. The vesicles were obtained using different sorbitan esters (Span 20, Span 40, and Span 60) and cholesterol. The results showed that these niosomes can be used as drug delivery carriers for targeting of rofecoxib to the skin. The niosomal gel allowed prolonged drug release, thereby sustaining the action of rofecoxib and reducing its adverse effects. Ketoprofen has also been encapsulated in niosomes containing Span 60 for topical application, and was released in a slow and sustained manner. ${ }^{43}$

A new transdermal formulation of tenoxicam, characterized by improved safety and high therapeutic efficacy, was developed by Ammar et $\mathrm{al}^{44}$ who designed a promising proniosome gel formulation intended to reduce the daily dose of drug that needed to be administered, in an effort to improve patient compliance. In this study, different proniosomal gel bases were prepared, characterized, and assessed for their drug entrapment efficiency, stability, effect on in vitro drug release, and ex vivo drug permeation. This tenoxicam-loaded proniosomal formulation proved to be nonirritant, with significantly higher anti-inflammatory and analgesic activity when compared with the oral tenoxicam tablets on the market.

Flurbiprofen was formulated as a proniosomal transdermal gel with high drug loading $(55.4 \%, \mathrm{w} / \mathrm{w})$ using a series of nonionic surfactants (Span 20, Span 40, and Span 60) and cholesterol. ${ }^{45}$ Data published by Zidan and Mokhtar ${ }^{45}$ revealed that these proniosomes afforded high drug loading and skin permeation. In particular, maximum permeation values and minimum encapsulation efficiency were obtained by the formulation containing Span with a shorter fatty acid chain length and a minimum amount of cholesterol.
A novel sustained-release proniosomal system was designed by El-Laithy et al using sugar esters as nonionic surfactants in which proniosomes were converted to niosomes upon hydration of the skin following topical application under occlusive conditions. ${ }^{46}$ The vesicles were loaded with vinpocetine, a potent anti-inflammatory agent that might also have a potential role in the treatment of Parkinson's disease and Alzheimer's disease. A number of in vitro parameters were studied, leading to an optimized formula that was assessed clinically for transdermal pharmacokinetics and skin irritation. All formulations prepared showed high entrapment efficiency and all vesicles were in a size range that favored efficient transdermal drug delivery. Taking into account the high efficiency of systemic delivery together with the lack of irritation and excellent safety profile, laurate sugar ester proniosomes could be considered as very promising candidates for enhancing the transdermal absorption and penetration of vinpocetine.

Niosomes loaded with baclofen, a centrally acting muscle relaxant, were prepared to improve the low skin penetration and poor bioavailability of conventional topical formulations containing this drug. ${ }^{47}$ Vesicles were prepared by the lipid film hydration method using nonionic surfactant (Span 20) and cholesterol in different molar ratios. Vehiculation of baclofen by niosomal formulations resulted in improved muscle relaxant activity.

Span 40-based niosomes were used as nanocarriers to improve percutaneous absorption of salidroside, a phenylpropanoid glycoside extracted from the root of Rhodiola rosea, known for its pharmacological properties. ${ }^{48}$ Enhanced permeation and skin deposition of salidroside were obtained, due to the effects of the excipients used in the preparation of the niosomes. Salidroside-loaded niosomes showed good biocompatibility with skin tissue, immortal human epidermal keratinocytes, and human embryonic skin fibroblasts, and in vitro cellular uptake experiments confirmed that these different types of skin cells had various channels for internalization of the nonionic surfactant vesicles.

Capsaicin is readily absorbed from the skin upon topical administration. Several capsaicin-based creams and patches are available as over-the-counter medications, usually contain the drug at concentrations of $0.025 \%-1 \%$, and are commonly used for pain relief. Niosomal carriers have been proposed for transdermal delivery of capsaicin. Vesicles were prepared using a defined ratio of Span 80 and Tween 80 in order to obtain systems with a specific hydrophilelipophile balance $(10,12$, and 14) and were characterized in terms of dimension, morphology, and drug entrapment 
efficiency. ${ }^{49}$ Niosomes were compared with microemulsions prepared from the same surfactants to reach a similar hydrophile-lipophile balance. Data obtained by Tavano et $\mathrm{al}^{49}$ revealed that better percutaneous capsaicin permeation was achieved with niosomes when compared with microemulsion-based formulations. This can be explained by the fact that the microemulsions were considerably more viscous than the niosomal suspensions, so that capsaicin diffused faster from the niosomal matrix than from the microemulsions where the capsaicin molecule has to overcome the immiscible oil phase to reach the skin.

Moreover, niosomes based on Tween 60 and containing resveratrol, alpha-tocopherol, and curcumin as single agents and in combination were designed with the aim of developing novel cosmeceutical products. ${ }^{50}$ The results showed that coencapsulation of resveratrol/curcumin and alpha-tocopherol/ curcumin affected the physicochemical properties of niosomes and the entrapment efficiency values when compared with the formulations containing the single antioxidant. The in vitro percutaneous permeation profiles for the antioxidants appeared to be controlled and improved with respect to the corresponding free solutions used as controls. In addition, the combination of antioxidants resulted in an improved ability to reduce free radicals due to a synergistic antioxidant action. Inhibition of free radicals was up to $40 \%$ for the resveratrol/ curcumin formulation, with the best performance being achieved by the alpha-tocopherol/curcumin sample (about $100 \%$ inhibition). However, the resveratrol/curcumin samples showed optimal performance in terms of cumulative amount of antioxidants permeated across the skin.

Papain is a protease enzyme from Carica papaya latex, which is widely used in dermatology for the treatment of scars. Manosroi et $a^{51}$ compared the transdermal release of papain from gel formulations containing niosomes or nanospheres. They demonstrated that niosomes (especially elastic niosomes obtained from Tween 61/cholesterol with sodium cholate as an edge activator) could enhance transdermal absorption of papain through rat skin and improve scar reduction in a rabbit ear model, which would be beneficial for development of topical products for the treatment of scars.

Simvastatin is a lipid-lowering agent used in the treatment of hypercholesterolemia in humans and animals. When administered orally, simvastatin undergoes extensive first-pass metabolism in the liver, resulting in low bioavailability and a short half-life of only 2 hours. A proniosomal system for transdermal delivery of simvastatin was developed by Shaker et $\mathrm{al}^{52}$ and subjected to in vitro and in vivo characterization. The results suggested that application of simvastatin-loaded proniosomes (composed of lecithin and Tween in a ratio of 1:9) onto the skin significantly improved not only the bioavailability of the drug but also its hypocholesterolemic effect in the treatment of hypercholesterolemic rats.

The prevalence of childhood dyslipidemia is increasing and is considered to be an important risk factor for cardiovascular disease in adulthood. To improve dosing accuracy and facilitate the determination of dosing regimens based on body weight, Zidan et $\mathrm{al}^{53}$ prepared a transdermal niosomal gel containing simvastatin as a possible transdermal drug delivery system for pediatric application. Transdermal administration of this niosomal gel in rats achieved a significant reduction in cholesterol and triglyceride levels and an increase in plasma high-density lipoprotein concentrations. These data show that this transdermal niosomal system not only has good potential for enhancing drug bioavailability and hypolipidemic activity, but could also be considered a promising antihyperlipidemic formulation for pediatric use.

More recently, El Maghraby et $\mathrm{a}^{54}$ investigated the effect of incorporation of skin penetration enhancers in proniosomes on the ability of this system to deliver drugs transdermally. To achieve this objective, nisoldipine, a second-generation dihydropyridine calcium antagonist, was selected as the model drug, based on the fact that nisoldipine suffers from low and variable bioavailability after oral administration because of extensive first-pass metabolism in the liver. Lecithin, oleic acid, and propylene glycol were used as enhancers to optimize transdermal delivery of nisoldipine from this provesicular system and to design a new methodology to enhance its transdermal delivery. According to the published report, all proniosomal formulations delivered the drug through the skin at a significantly higher rate when compared with the saturated aqueous control formulation and the enhancing efficacy of the oleic acid/propylene glycol combination was even better than that of lecithin.

Nifedipine is a calcium channel blocker used extensively in the treatment of angina and hypertension. After oral administration, it undergoes extensive first-pass metabolism, which limits both the amount of drug absorbed in the gastrointestinal tract and the bioavailability of the drug in the systemic circulation. Yasam et $\mathrm{a}^{55}$ proposed the transdermal route as an alternative method of administration for nifedipine. In this study, proniosomes were prepared by varying the ratios of Span 40, lecithin, aqueous phase, and polymer. Ex vivo percutaneous permeation studies in rat skin revealed the role of niosomes as permeation enhancers. Moreover, skin irritation studies showed that when loaded into proniosomes, 
nifedipine was not irritant and did not cause erythema, unlike the pure drug.

There has been a report of transdermal delivery of niosomes loaded with sulfadiazine sodium, an antibiotic usually used for the topical treatment of infected burns. ${ }^{56}$ In this study, the effects of inclusion of different amounts of ethanol, propylene glycol, and glycerol in the niosomes were specifically evaluated. The presence of alcohol critically affected the physicochemical properties of niosomes, ie, vesicular size increased with amounts of alcohol, and at the same alcohol concentration, bigger vesicles were obtained with ethanol, followed by propylene glycol, and glycerol. Comparison of the percutaneous drug permeation profiles showed that the cumulative amount of permeated sulfadiazine sodium increased with alcohol concentration, up to $20 \% \mathrm{v} / \mathrm{v}$; a higher concentration $(40 \% \mathrm{v} / \mathrm{v})$ resulted in a strong decrease in skin permeation.

Novel multicomponent formulations were studied by Tavano et $\mathrm{al}^{35}$ to evaluate the effect of the chemical structure of several surfactants and vehiculated drug on the physicochemical properties of the carriers and on percutaneous drug permeation profiles. Pluronic L64 or Aerosol OT were used as surfactants, and sulfadiazine sodium salt, propranolol hydrochloride, and tyrosol were chosen as the model drugs. Lyotropic liquid crystals were prepared at a fixed ratio between Pluronic L64 or Aerosol OT and water, in order to obtain lamellar lyotropic liquid crystal phases, and the corresponding surfactant was used to prepare the vesicular systems (niosomes) that were added to the gel. All the studied formulations, such as gel-niosome systems, niosomal suspensions and gels, act as percutaneous permeation enhancers respect to the corresponding free drug solutions used as control.

Another interesting study was reported by Kong et $\mathrm{al}^{57}$ in which functionalized niosomes were used as a transdermal drug delivery system for tumor therapy. These investigators have developed a novel drug nanocarrier based on hyaluronic acid and niosomes, which promotes transdermal drug delivery and has tumor-targeting ability. An amphiphilic hyaluronic acid esterified with glycerol monostearate was prepared and self-assembled onto the niosome surface to give hyaluronic acid niosomes. Their results showed that incorporation of hyaluronic acid significantly promoted endocytosis of the nanocarriers into tumor cells. Hyaluronic acid niosomes may be considered not only as efficient and safe carriers for transdermal permeation, but may also represent a useful and promising device for tumor therapy through percutaneous administration.
Lopinavir, a highly potent protease inhibitor used in the treatment of AIDS, needs to be coadministered with ritonavir because of its high presystemic metabolism, which results in poor systemic bioavailability. In a study reported by Patel et al, ${ }^{34}$ a niosomal gel was developed to improve the systemic availability of lopinavir via the transdermal route. The niosomal formulation containing lopinavir, Span 40, and cholesterol contained a high amount of loaded drug with a minimum mean vesicular diameter. Encouraging results in terms of niosomal stability, skin permeability, lack of irritation, and ability to improve the overall bioavailability of lopinavir led to the conclusion that niosomal gel has considerable promise as a novel transdermal nanocarrier for safe delivery of lopinavir into the systemic circulation.

Despite the large amount of research published in recent decades, very few studies on topical niosomal formulations and their possible applications have progressed to patents and preclinical or clinical trials. Proniosomal gels loaded with finasteride ${ }^{58}$ have undergone transdermal pharmacokinetic evaluation, while urea ${ }^{59,60}$ and griseofulvin ${ }^{61}$ niosomal gels have reached the stage of clinical trials. Lancome and L'Oreal have also developed a variety of antiaging products based on niosomal formulations.

\section{Conclusion and future perspectives}

This review offers a comprehensive explanation of the chemical composition, structure, advantages, and applications of niosomes, their role as percutaneous permeation enhancers, and their recent applications as delivery systems for transdermal drug targeting. Niosomes have been demonstrated to be promising controlled delivery systems for percutaneous administration of both hydrophilic and lipophilic drugs. The potential of niosomes can be enhanced by using novel preparation, loading, and modification methods. These areas need further exploration and research for the development of niosomal preparations that can be made commercially available. Researchers should be alert in the need for appropriate selection of suitable surfactants for preparation of niosomes, given that the type of surfactant used is the main parameter determining the successful formation of these vesicles, along with their toxicity, stability, and potential applications.

\section{Disclosure}

The authors report no conflicts of interest in this work.

\section{References}

1. Uchegbu IF, Florence AT. Nonionic surfactant vesicles (niosomes): physical and pharmaceutical chemistry. Adv Colloid Interface Sci. 1995;58:1-55 
2. Moghassemi S, Hadjizadeh A. Nano-niosomes as nanoscale drug delivery systems: an illustrated review. J Control Release. 2014;185: 22-36.

3. Lasic DD. On the thermodynamic stability of liposomes. J Colloid Interface Sci. 1990;140:302-304.

4. Gannu PK, Rajeshwarrao P. Nonionic surfactant vesicular systems for effective drug delivery an overview. Acta Pharmacol Sin. 2011; 1 : 208-219.

5. Mahale NB, Thakkar PD, Mali RG, Walunj DR, Chaudhari SR. Niosomes: novel sustained release nonionic stable vesicular systems an overview. Adv Colloid Interface Sci. 2012;183:46-54.

6. Obata Y, Takayama K, Maitani Y, Machida Y, Nagai T. Effect of ethanol on skin permeation of nonionized and ionized diclofenac. Int J Pharm. 1993;89:191-198.

7. Perche F, Torchilin VP. Recent trends in multifunctional liposomal nanocarriers for enhanced tumor targeting. J Drug Deliv. 2013; 2013: 705265.

8. Pawar SD, Pawar RG, Kodag PP, Waghmare AS. Niosome: an unique drug delivery system. International Journal of Biology, Pharmacy and Allied Sciences. 2012;3:406-416.

9. Khan A, Sharma PK, Visht S, Malviya R. Niosomes as colloidal drug delivery system: a review. Journal of Chronotherapy and Drug Delivery. 2011;2:15-21.

10. Florence AT, Baillie AJ. Nonionic surfactant vesicles - alternatives to liposomes. In: Prescott LF, Nimmo WS, editors. Novel Drug Delivery and its Therapeutic Application. New York, NY, USA: John Wiley and Sons Ltd; 1989.

11. Thakur V, Arora S, Prashar B, Vishal P. Niosomes and liposomes vesicular approach towards transdermal drug delivery. International Journal of Pharmaceutical and Chemical Sciences. 2012;1: 981-993.

12. Nasr M, Mansour S, Mortada ND, Elshamy AA. Vesicular aceclofenac systems: a comparative study between liposomes and niosomes. J Microencapsul. 2008;25:499-512.

13. Handjani-Vila RM, Riber A, Rondot B, Valenberghe G. Aqueous dispersion. Int J Cosmet Sci. 1979;1:303-314.

14. Barel A, Paye M, Maibach HI. Handbook of Cosmetic Science and Technology. New York, NY, USA: Marcel Dekker Inc.; 2001.

15. Singh S. Niosomes: a role in targeted drug delivery system. Int J Pharm Sci Res. 2013;4:550-557.

16. Vyas SP, Khar RK. Novel carrier systems. In: Jain NK, editor. Targeted and Controlled Drug Delivery. New Delhi, India: CBS Publishers and Distributors Pvt Ltd; 2010.

17. Sahin NO. Niosomes as nanocarrier systems. In: Mozafari MR, editor. Nanomaterials and Nanosystems for Biomedical Applications. New York, NY, USA: Springer; 2007.

18. Hofland HE, Bouwstra JA, Verhoef JC, et al. Safety aspects of non-ionic surfactant vesicles: a toxicity study related to the physicochemical characteristics of non-ionic surfactants. J Pharm Pharmacol. 1992; 44: 287-294.

19. Pape WJ, Pfannenbecker U, Hoppe U. Validation of the red blood cell test system as in vitro assay for the rapid screening of irritation potential of surfactants. Mol Toxicol. 1987;1:525-536.

20. Tavano L, Infante MR, Abo Riya M, et al. Role of aggregate size in the hemolytic and antimicrobial activity of colloidal solutions based on single and gemini surfactants from arginine. Soft Matter. 2013;9: 306-319.

21. Paolino D, Muzzalupo R, Ricciardi A, Celia C, Picci N, Fresta M. In vitro and in vivo evaluation of bola-surfactant containing niosomes for transdermal delivery. Biomed Microdevices. 2007;9:421-433.

22. Walker RB, Smith EW. The role of percutaneous penetration enhancers. Adv Drug Deliv Rev. 1996;18:295-301.

23. Barry BW. Lipid protein partitioning theory of skin penetration enhancement. J Control Release. 1991;15:237-248.

24. Schreier H, Bouwstra J. Liposomes and niosomes as topical drug carriers: dermal and transdermal drug delivery. J Control Release. 1994; $30: 1-15$.
25. Karande P, Mitragotri S, Enhancement of transdermal drug delivery via synergistic action of chemicals. Biochim Biophys Acta. 2009; 1788: 2362-2373.

26. Bouwstra JA, Honeywell-Nguyen PL. Skin structure and mode of action of vesicles. Adv Drug Deliv Rev. 2002;54:41-55.

27. Muzzalupo R, Tavano L, Cassano R, Trombino S, Ferrarelli T, Picci N. A new approach for the evaluation of niosomes as effective transdermal drug delivery systems. Eur J Pharm Biopharm. 2011;79:28-35.

28. Manconi M, Sinico C, Valenti D, Lai F, Fadda AM. Niosomes as carriers for tretinoin. III. A study into the in vitro cutaneous delivery of vesicle-incorporated tretinoin. Int J Pharm. 2006;27:11-19.

29. Abdelkader H, Alani AW, Alany RG. Recent advances in non-ionic surfactant vesicles (niosomes): self-assembly fabrication, characterization, drug delivery applications and limitations. Drug Deliv. 2014;21: 87-100.

30. Mali N, Darandale S, Vavia P. Niosomes as a vesicular carrier for topical administration of minoxidil: formulation and in vitro assessment. Drug Deliv Transl Res. 2013;3:587-592.

31. Cevc G. Lipid vesicles and other colloids as drug carriers on the skin. Adv Drug Deliv Rev. 2004;56:675-711.

32. El Maghraby GM, Barry BW, Williams AC. Liposomes and skin: from drug delivery to model membranes. Eur J Pharm Sci. 2008;34: 203-222.

33. Touitou E, Dayan N, Bergelson L, Godin B, Eliaz MJ. Ethosomes novel vesicular carriers for enhanced delivery: characterization and skin penetration properties. Control Release. 2000;65:403-418.

34. Patel KK, Kumar P, Thakkar HP. Formulation of niosomal gel for enhanced transdermal lopinavir delivery and its comparative evaluation with ethosomal gel. AAPS Pharm Sci Tech. 2012;13:1502-1510.

35. Tavano L, Gentile L, Oliviero Rossi C, Muzzalupo R. Novel gel-niosome formulations as multicomponent systems for transdermal drug delivery. Colloids Surf B Biointerfaces. 2013;110:281-288.

36. Alsarra IA, Bosela AA, Ahmed SM, Mahrous GM. Proniosomes as a drug carrier for transdermal delivery of ketorolac. Eur J Pharm Biopharm. 2005;59:485-490.

37. Solanki AB, Parikh JR, Parikh RH, Patel MR. Evaluation of different compositions of niosomes to optimize aceclofenac transdermal delivery. Asian Journal of Pharmaceutical Sciences. 2010;5:87-95.

38. El-Menshawe SF, Hussein AK. Formulation and evaluation of meloxicam niosomes as vesicular carriers for enhanced skin delivery. Pharm Dev Technol. 2013;18:779-786.

39. El-Badry M, Fetih G, Fathalla D, Shakeel F. Transdermal delivery of meloxicam using niosomal hydrogels: in vitro and pharmacodynamic evaluation. Pharm Dev Technol. 2014;9:1-7.

40. Manosroi A, Jantrawuta P, Manosroi J. Anti-inflammatory activity of gel containing novel elastic niosomes entrapped with diclofenac diethylammonium. Int J Pharm. 2008;360:156-163.

41. Tavano L, de Cindio B, Picci N, Ioele G, Muzzalupo R. Drug compartmentalization as strategy to improve the physico-chemical properties of diclofenac sodium loaded niosomes for topical applications. Biomed Microdevices. 2014;16:851-858.

42. Das MK, Palei NN. Sorbitan ester niosomes for topical delivery of rofecoxib. Indian J Exp Biol. 2011;49:438-445.

43. Rajnish A, Ajay S. Release studies of ketoprofen niosome. J Chem Pharm Res. 2010;1:79-82.

44. Ammar HO, Ghora M, El-Nahhas SA, Higazy IM. Proniosomes as a carrier system for transdermal delivery of tenoxicam. Int J Pharm. 2011;405:142-152.

45. Zidan AS, Mokhtar M. Multivariate optimization of formulation variables influencing flurbiprofen proniosomes characteristics. J Pharm Sci. 2011;100:2212-2221.

46. El-Laithy HM, Shoukry O, Mahran LG. Novel sugar esters proniosomes for transdermal delivery of vinpocetine: preclinical and clinical studies. Eur J Pharm Biopharm. 2011;77:43-55.

47. Keservani RK, Sharma AK, Ramteke S. Novel vesicular approach for topical delivery of baclofen via niosomes. Lat Am J Pharm. 2010; 29: 1364-1370. 
48. Zhang Y, Zhang K, Wu Z, et al. Evaluation of transdermal salidroside delivery using niosomes via in vitro cellular uptake. Int J Pharm. 2015; 478:138-146.

49. Tavano L, Alfano P, Muzzalupo R, de Cindio B. Niosomes vs microemulsions: new carriers for topical delivery of capsaicin. Colloids Surf B Biointerfaces. 2011;87:333-339.

50. Tavano L, Muzzalupo R, Picci N, de Cindio B. Co-encapsulation of lipophilic antioxidants into niosomal carriers: Percutaneous permeation studies for cosmeceutical applications. Colloids Surf B Biointerfaces. 2014;114:114-149.

51. Manosroi A, Chankhampan C, Manosroi W, Manosroi J. Transdermal absorption enhancement of papain loaded in elastic niosomes incorporated in gel for scar treatment. Eur J Pharm Sci. 2013;48:474-483.

52. Shaker DS, Nasr M, Mostafa M. Bioavailability and hypocholesterolemic effect of proniosomal simvastatin for transdermal delivery. International Journal of Pharmacy and Pharmaceutical Sciences. 2013; 5:344-351.

53. Zidan AS, Hosny KM, Ahmed OA, Fahmy UA. Assessment of simvastatin niosomes for pediatric transdermal drug delivery. Drug Deliv. 2014;11:1-14

54. El Maghraby GM, Ahmed AA, Osman MA. Penetration enhancers in proniosomes as a new strategy for enhanced transdermal drug delivery. Saudi Pharm J. 2015;23:67-74.
55. Yasam VR, Jakki SL, Natarajan J, et al. Novel vesicular transdermal delivery of nifedipine - preparation, characterization and in vitro/invivo evaluation. Drug Deliv. 2014;9:1-12.

56. Muzzalupo R, Tavano L, Lai F, Picci N. Niosomes containing hydroxyl additives as percutaneous penetration enhancers: effect on the transdermal delivery of sulfadiazine sodium salt. Colloids Surf B Biointerfaces. 2014;123:207-212.

57. Kong M, Park H, Feng C, Hou L, Cheng X, Chen X. Construction of hyaluronic acid niosome as functional transdermal nanocarrier for tumor therapy. Carbohydr Polym. 2013;94:634-641.

58. Kassem MA, Esmat S, Bendas ER, El-Komy MH. Efficacy of topical griseofulvin in treatment of tinea corporis. Mycoses. 2006;49: 232-235.

59. Lakshmi PK, Bhaskaran S. Phase II study of topical niosomal urea gel - an adjuvant in the treatment of psoriasis. International Journal of Pharmaceutical Sciences Review and Research. 2011;7:1-7.

60. Mazda F, Özer AY, Ercan MT. Preparation and characterisation of urea niosomes - in vitro and in vivo studies. STP Pharm Sci. 1997;7: 205-214.

61. Ribeiro de Souza AL, Kiill CP, Kolenyak dos Santos FK, et al. Nanotechnology-based drug delivery systems for dermatomycosis treatment. Curr Nanosci. 2012;8:512-519.
Research and Reports in Transdermal Drug Delivery

\section{Publish your work in this journal}

Research and Reports in Transdermal Drug Delivery is an international, peer-reviewed, open access online journal publishing original research, study protocols, reviews, editorials and commentaries on all aspects of transdermal drug delivery. Specific topics in the journal include: Laboratory and clinical development of drug delivery systems including preclinical, clinical studies and protocols; Rationale and basic science; Drug

\section{Dovepress}

delivery via gels, creams or patches; Use of chemical drug penetration enhancers ; Patient acceptability studies; and pharmacoeconomic and clinical outcome studies. The manuscript management system is completely online and includes a very quick and fair peer-review system, which is all easy to use. Visit http://www.dovepress.com/ testimonials.php to read real quotes from published authors.

Submit your manuscript here: http://www.dovepress.com/research-and-reports-in-transdermal-drug-delivery-journal 